\title{
Relación entre estilos de aprendizaje y rendimiento académico de estudiantes de Licenciatura en Pedagogía Infantil-UNIMINUTO ${ }^{1}$
}

\author{
The relation between learning styles and academic \\ performance of the bachelor degree in Child \\ Pedagogy-UNIMINUTO students
}

\author{
Ada Marcela González Riveros ${ }^{2}$ \\ Carlos Mauricio Salamanca Bahamón ${ }^{3}$
}

\section{Resumen}

La presente investigación reflexiona en torno a la diversidad de estilos de aprendizaje de las estudiantes del módulo de desarrollo de la Licenciatura en Pedagogía infantil perteneciente a la Facultad de Educación de la Corporación Universitaria Minuto de Dios. El propósito del estudio se centró en desvelar la correlación entre los estilos de aprendizaje y el rendimiento académico de una muestra conformada por 75 estudiantes entre 18 y 25 años. La metodología utilizada fue de tipo cuantitativo con un diseño expos-facto, donde se aplicó el cuestionario Honey-Alonso de estilos de aprendizaje- CHAEA (1994) a estudiantes de tres espacios académicos y se correlacionó con los resultados de las valoraciones finales de estos. Los resultados indican que las estudiantes de estos grupos que desarrollan un estilo de aprendizaje reflexivo o pragmático tienden a obtener valoraciones altas o superiores en una escala numérica, mientras que las que desarrollan estilos activos o teóricos presentan una dispersión académica amplía en sus valoraciones.

Palabras clave: Estilos de aprendizaje; Rendimiento académico; Licenciaturas.

\begin{abstract}
The following research work is a reflection ab out the diversity of learning styles of students studying the module development that is part of the bachelor degree program in Child pedagogy of the Faculty of Education from Universidad Minuto de Dios. The purpose of the study was to show the relation between learning styles and academic performance of a group of seventy-five students between the ages of 18 and 25 . For this study a quantitative methodology with an expos-facto design was applied using the learning style Honey-Alonso test CHAEA (1994), it was applied to the students of three different academic sectors and it was correlated with the results of the final evaluations. The results show that the students of the groups who develop a reflexive and pragmatic learning style tend to get high results whereas those who develop an active and theoretical learning styles show a wide academic dispersion in their results.
\end{abstract}

Keywords: Learning styles; Academic performance; Bachelor degrees.

\footnotetext{
${ }^{1}$ Artículo de investigación derivado del proyecto "Influencia de las inteligencias múltiples y los estilos de cognoscitivos en la utilización de estrategias de aprendizaje" 2015. ${ }^{2}$ Magister en Educación Pontificia Universidad Javeriana y Magíster en Neuropsicología y Educación Universidad Internacional de la Rioja España (2016). Docente de la Corporación universitaria Minuto de Dios. E-mail amgonzalez@uniminuto.edu

${ }^{3}$ Licenciado en Electrónica Universidad Pedagógica Nacional (2012) Docente de la Corporación universitaria Minuto de Dios. E-mail carlos.salamanca@uniminuto.edu
} 


\section{Introducción}

El acceso a la Educación Superior requiere de la maduración de habilidades y estrategias que permitan un desempeño académico óptimo, acorde a las demandas del contexto universitario y que, a su vez, faciliten la adquisición de competencias genéricas y específicas propias del campo en el que ejercerán su praxis. En este sentido, la preparación de profesionales para la educación, encierra en sí misma una gran responsabilidad social por parte de las instituciones universitarias y de los docentes que tienen a cargo la formación de los nuevos educadores. Al respecto, los estudios de Gallego y García (2012), Cardona (2005) y Mendoza (2012) entre otros, coinciden que la calidad educativa aumenta al trabajar en estrategias que cualifiquen los procesos de enseñanza y aprendizaje en las universidades y en específico en las facultades de educación, sumado a ello, De Tezanos (2006) expresa que "la calidad de la enseñanza en las escuelas y liceos emerge estrechamente vinculada a los modos como se asume la formación de docentes" (p. 25)

Ante este panorama, los programas de formación de maestros deben tener en cuenta los factores que influyen en la adquisición de dichas competencias, con el fin de aproximarse a la comprensión de las diversas formas de conocer y producir nuevos conocimientos. En este punto, surgen dos elementos a considerar: primero, los procesos educativos a nivel universitario son responsabilidad de docentes y estudiantes, por lo cual, el aprendizaje es un acto conjunto de retroalimentación, mediación e interacción que supone reflexiones en el aula y que requiere de procedimientos, técnicas y estrategias que lleven al éxito de unos y otros. Segundo, la valoración de la diversidad en las aulas inicia desde el reconocimiento de las características del contexto donde se desenvuelve el sujeto, hasta las variables de orden interno asociadas al desarrollo genético, cognitivo, neurológico, psicológico, biológico entre otros.

En este orden de ideas, se hace necesario que las licenciaturas incorporen instrumentos para la comprensión de los estilos de aprendizaje que se presentan en los maestros en formación y la relación de estos en un contexto particular de formación, es decir, que el reconocimiento de los estilos incite a los docentes a reflexionar sobre las características de la población, los ritmos de aprendizaje, las metodologías y las maneras de evaluación que se implementan en el aula. Así mismo, que se amplíe la concepción construida de aprendizaje y su respectiva valoración por medio de las escalas numéricas que se asocian al rendimiento académico de los estudiantes.

Conforme a lo dicho, surgió el interés por parte de los docentes investigadores por indagar sobre los estilos de aprendizaje que comporta una muestra de estudiantes de $\mathrm{V}$ semestre del programa de Licenciatura en Pedagogía Infantil-LPIN de la Corporación universitaria Minuto de Dios-UNIMINUTO y su respectiva correlación con el rendimiento académico obtenido en los espacios académicos del módulo de desarrollo. El proyecto que respalda este texto planteó dentro de sus objetivos la identificación de los estilos de aprendizaje por medio del cuestionario CHAEA (Alonso et al., 1994) y el rendimiento académico tomando como base la valoración final de las asignaturas desarrollo cognitivo y comunicativo, desarrollo corporal y desarrollo ético pertenecientes al módulo de desarrollo de la LPIN, el resultado cuantitativo o nota es igual para todas las materias que lo conforman.

El módulo hace parte del área curricular denominada "Desarrollo infantil", la cual pretende que las docentes en formación comprendan el 
desarrollo infantil desde una mirada holística que permita analizar situaciones y diseñar propuestas pedagógicas pertinentes a las infancias de diferentes contextos. En este sentido, el alcance de esta competencia requiere de la conjunción de capacidades teórico-prácticas enfocadas al proceso bidireccional de enseñanza-aprendizaje del desarrollo sensorial, perceptivo, motor, lingüístico y de pensamiento; todo ello con el fin de aportar a la construcción de ambientes de aprendizaje basados en los principios de arte, juego, literatura y exploración del medio. Las estrategias metodológicas que estructuran el microcurriculo del módulo, hacen uso de estudios de caso, simulación de clase, talleres, elaboración de proyectos, Aprendizaje Basado en resolución de Problemas - ABP- y clases magistrales distribuidas en espacios presenciales y virtuales.

La importancia del estudio radica en el interés que tienen los docentes investigadores por comprender y cualificar las prácticas docentes desarrolladas en el marco de un modelo universitario inclusivo como el que caracteriza a UNIMINUTO y lograr así una verdadera transformación de las estrategias de enseñanza en el aula. En otras palabras, conocer los diferentes estilos de aprendizaje que confluyen en el aula facilita la transformación del pensamiento docente a través de experiencias colectivas de creación de nuevos ambientes de aprendizaje que privilegien las potencialidades de docentes y estudiantes al desarrollar las estrategias metodológicas, aspecto que se puede ver reflejado en su ejercicio profesional como licenciadas. Si bien es cierto, la gestión de una propuesta educativa en el marco de la diversidad, en palabras de Escudero (1990, citado en Arnaiz 2003) involucra una estructura organizativa que promueva la educación para todos, un currículo con estrategias educativas acordes a la población, profesionales con actitud de cambio y la comprensión de procesos de enseñanza y aprendizaje basados en la experiencia individual, las capacidades y la interacción como principio. Esta investigación nutre a la línea de investigación de procesos de memoria y habilidades de pensamiento del Máster de Neuropsicología y Educación.

\section{Una aproximación conceptual a los estilos de aprendizaje y su relación con el rendimiento académico}

La diversidad presente en los seres humanos conlleva a repensar la comprensión de los procesos educativos a la luz de los campos científicos y en específico ¿cómo estos afectan? en las relaciones que se gestan entre estudiantes y maestros en las aulas de cualquier nivel educativo. Al respecto, disciplinas como la psicología y neurología, expresan que la unidad funcional que tienen los seres humanos es la misma, pero al someterse a las variadas experiencias que vive el sujeto en el medio, se constituyen los aspectos diferenciales o estilos de cada ser.

Al hablar de los estilos, es importante destacar que se usa el término según el perfil teórico o disciplina que los estudia, en el campo educativo se prefiere nominarlos como estilo de aprendizaje y en el campo psicológico los llaman estilos cognoscitivos, sin embargo sea una forma u otra, lo que enmarca las investigaciones en este tema es la preocupación por comprender, identificar y utilizar las formas de aprender para obtener un mejor provecho en beneficio personal y social (Sánchez y Andrade, 2014). Para mayor comprensión en la Tabla.1 se recopilan diferentes definiciones de los estilos cognoscitivos o de aprendizaje más relevantes en la literatura científica: 
Tabla 1: diferentes definiciones de los estilos cognoscitivos o de aprendizaje

\begin{tabular}{|c|l|}
\hline Keefe (1988) & $\begin{array}{l}\text { Los rasgos cognitivos, afectivos } \\
\text { y fisiológicos, que sirven como } \\
\text { indicadores relativamente estables, } \\
\text { de cómo los alumnos perciben, } \\
\text { interaccionan y responden a los } \\
\text { ambientes de aprendizaje }\end{array}$ \\
\hline Reinert, (1976) & $\begin{array}{l}\text { Es la manera por la cual una persona } \\
\text { es programada para aprender lo más } \\
\text { eficientemente, es decir, para recibir, } \\
\text { comprender, memorizar y ser capaz } \\
\text { de utilizar la nueva información. }\end{array}$ \\
\hline Gregory (1979) & $\begin{array}{l}\text { El estilo de aprendizaje consiste en } \\
\text { comportamientos distintivos que sirven } \\
\text { como indicadores de cómo una } \\
\text { persona aprende y se adapta a su } \\
\text { ambiente. }\end{array}$ \\
\hline Kerby, Estwtbe y & $\begin{array}{l}\text { Conjunto de estrategias que abarcan } \\
\text { no solo cuestiones puramente } \\
\text { cognitivas, sino también las que } \\
\text { denominan de apoyo como la } \\
\text { motivación o la autoestima. }\end{array}$ \\
\hline Adán (2001) & $\begin{array}{l}\text { Son procedimientos generales } \\
\text { de aprendizaje integrados por } \\
\text { componentes cognoscitivos, afectivos } \\
\text { y conductuales que empleamos de } \\
\text { forma diferenciada para resolver } \\
\text { situaciones de aprendizaje en } \\
\text { diferentes contextos. }\end{array}$ \\
\hline
\end{tabular}

Fuente: Elaboración propia (2015) basada en Aguilera \&Torres (2009)

En este sentido, se comprende que el acto de aprender involucra un estilo propio, que responde a las preferencias que tienen los individuos al percibir, procesar y representar la información. Según (Alonso, Gallego y Honey 1995, citados en Salas, 2012, p 14) "los estilos son algo así como conclusiones a las que llegamos acerca de la manera cómo actúan las personas" estos actos abarcan según (Santos R y Doval, 1993, citados en Salas, 2012) la cognición ¿Cómo se yo?, la conceptualización ¿Cómo pienso?, el afecto ¿Cómo siento y reacciono? y con la conducta ¿Cómo actúo? Desde esta postura, resulta lógico pensar que los estudiantes pueden presentar afinidad por un conjunto de estrategias para aprender y adaptarse a la academia, pero ello no significa que se encapsulen según el estilo predominante detectado.

$\mathrm{Al}$ respecto, Bustos (s.f) afirma que "no se puede estereotipar o rotular a un estudiante por su proceso de aprendizaje, porque éste varía según sus intereses, sus estados emocionales o sus dinámicas de vida" ( $p$. 86). Lozano (2006) expresa que las situaciones influyen en los patrones comportamentales de los individuos, por lo cual un estilo no es definitivo, así como las relaciones entre logros o competencias y estilo no demarcan cuál es el mejor o peor. En este sentido, los docentes deben estar vigilantes y dar apertura a las posibilidades para enriquecer las estrategias de enseñanza co-construidas con los estudiantes y de esta forma suscitar un nuevo orden en las relaciones que se establecen en el aula, es decir, invita a entender las relaciones de enseñanza y aprendizaje desde un enfoque no lineal, causal o fragmentado. En palabras de Morin (2009) "Aunque la diversidad humana es visible (...) ha devenido hoy invisible para las mentes que no conocen más que troceando, separando, catalogando, compartimentando. O también, lo que aparece a las mentes abstractas es una unidad abstracta que oculta las diferencias" (p. 66) por lo cual, se comprende que la diversidad es inherente a la complejidad de lo humano.

En línea con lo anterior, Esguerra y Guerrero (2010) enuncian que el rendimiento académico no es un tema simple, pues está permeado por diferentes factores de distinto orden conformando una red compleja. Para los autores, existen cinco tipos de variables que estudian el fenómeno en diferentes contextos de formación:

Las variables de identificación (género, edad); las variables académicas (tipos de estudios cursados, curso, opción en que se estudia una carrera, rendimiento previo, etc.); las variables pedagógicas (definición de competencias de aprendizaje, metodología de enseñanza, estrategias de evaluación, etc.); las variables socio-familiares (estudios 
de los padres, profesión, nivel de ingresos, etc.) y las variables psicológicas (aptitudes intelectuales, personalidad, motivación, estrategias de aprendizaje, estilos de aprendizaje, entre otros). (p.101)

\section{Modelos representativos de estilos de aprendizaje}

Se han desarrollado modelos y tipologías asociadas a los estilos de aprendizaje que permiten aproximarse a la comprensión de la diversidad de procesos que se producen en un aula de clase. Para este trabajo se retomará el Modelo de Kolb (1970) y el de Honey y Mumford (1986), pues estos autores orientan la comprensión que el cuestionario CHAEA aplicado.

Según Salas (2012) el modelo de Kolb se basa en el análisis de las experiencias que propician el aprendizaje que pueden ser directas o abstractas. Dichas vivencias se transforman en conocimiento cuando las elaboramos: reflexionando, pensando sobre ellas o experimentando en forma activa con la información recibida. Para el autor, el aprendizaje es comprendido desde un ciclo evolutivo de cuatro estadios que incluyen cuatro modos adaptativos:

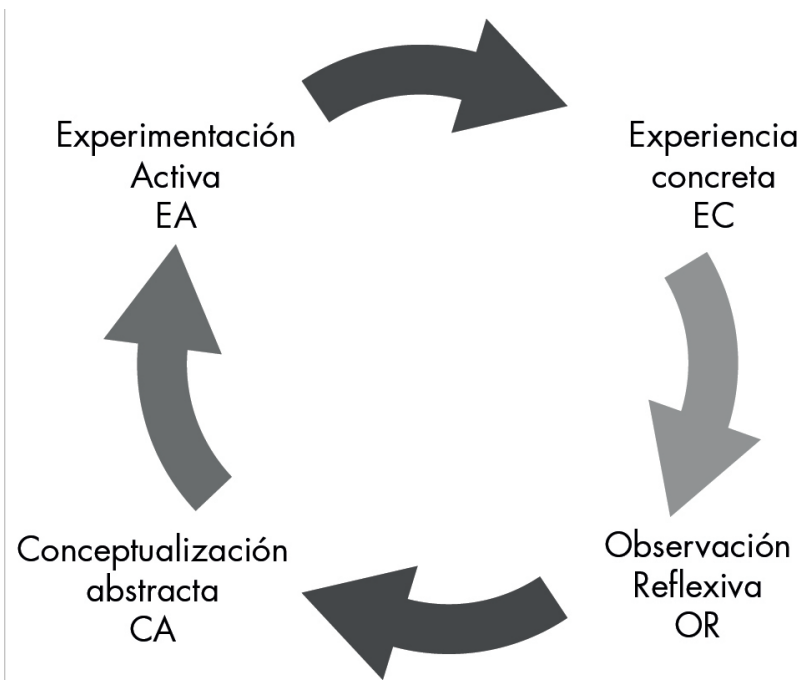

Figura 1. Modelo de Kolb
Las transiciones entre los cuatro modos y la forma en que interactúan las dialécticas adaptativas originan el proceso de aprendizaje, es decir que el conocimiento se crea por medio de la modificación de la experiencia, que en un primer plano es concreta, al hacer uso de los sentidos y sentimientos, seguido por la observación reflexiva que lleva a la conceptualización a partir de la lógica y, finalizando, con experimentación activa que se remite al aprender haciendo. Por lo tanto, es más fácil que durante el acto de aprender se combinen estas fases con el fin de aprovechar mejor la experiencia educativa.

En esta misma línea diferentes autores (Aguilera \& Torres 2009; Aguilar, 2010; Salas, 2008; Sánchez y Andrade 2014) exponen que los estilos de aprendizaje que plantea Kolb son:

- Activos o acomodadores: incluye competencias o habilidades para actuar o hacer, experimentan, indagan, actúan primero y reflexionan después, les gusta el trabajo en equipo. Su transformación viene dada por la experiencia activa y sensorial. No les agrada el papel pasivo o los periodos largos sin interacción o participación.

- Reflexivos o asimiladores: relacionado con las competencias para pensar, captan la experiencia abstractamente, son observadores, reflexivos, piensan antes de actuar, son seguros, planificados, juzgan y mediante el análisis de la información obtienen conocimiento. Se les dificulta aprender si están como líderes públicos, actuar, improvisar, requieren siempre estar sin tener datos 0 información.

- Teóricos o divergentes: asociados con el valorar, captan la información concreta y la transforman reflexivamente, metódicos, sintetizan información les gusta leer, resumir, anotar, resumir y comprobar sus 
razonamientos. Piensan secuencialmente integrando conocimientos teóricos, evitan los juicios subjetivos por lo cual buscan argumentación.

- Pragmáticos o convergentes: son personas que les gusta probar sus teorías en la práctica, relacionados con la habilidad de decisión, los convergentes captan la información abstractamente (reflexivos) y la procesan activamente (hacedores), requieren comprobar por sí mismos. Les gusta trabajar en equipo, son espontáneos, les agrada la improvisación, organizan actividades con rapidez previniendo consecuencias y resultados. Se les dificulta trabajar solos, callados, quietos, con instrucciones claras.

\section{Metodología}

La presente investigación, se centra en el método descriptivo (Hernández, Fernández y Baptista, 2010, p. 80) y se sustenta en el paradigma interpretativo, cuyo interés fue el de comprender la relación de estilos de aprendizaje (Honey \& Alonso, 1994) y la valoración académica de las estudiantes de Licenciatura en Pedagogía Infantil del módulo desarrollo de la Corporación Universitaria Minuto de Dios-UNIMINUTO, cuyo enfoque fue cuantitativo y de diseño expos-facto. Este estudio, se llevó a cabo durante cuatro meses (duración del semestre académico) compuesto por 16 sesiones divididas en 64 horas presenciales y 128 horas de trabajo autónomo para todo el modulo. Las estrategias metodológicas que se usaron, se centraron en el Aprendizaje Basado en resolución de Problemas -ABP-, estudio de caso, simulación de clase y proyectos.

El estudio se desarrolló con una población compuesta por las 75 estudiantes de V semestre que asisten al módulo de desarrollos que se imparte de manera independiente en las jornadas diurna y nocturna de la Licenciatura en Pedagogía Infantil de la Corporación Universitaria Minuto de Dios - UNIMINUTO sede principal Bogotá. El horario de estudio de la tarde es de 3:15 a 6:15 pm, en la noche es de 6:15 a 9:15 pm, las estudiantes de la jornada diurna se caracterizan por ser estudiantes solteras en su mayoría, no contar con vínculos laborales y vivir con sus padres; la muestra de la noche se caracteriza por tener compromisos laborales en el día relacionados en ocasiones con su carrera, ser madres cabeza de familia o tener vinculo de pareja con responsabilidades en la crianza de hijos. La edad varía en un rango de 18 a 24 años, tendiendo a mayores edades en la jornada nocturna. No existen criterios de exclusión y la muestra es femenina en su totalidad.

En primera instancia, se realizó una caracterización de la muestra, midiendo los estilos de aprendizaje de las estudiantes de la LPIN, con la ayuda del cuestionario CHAEA (Honey \& Alonso, 1994). Este cuestionario esta validado y ha sido aplicado en varias partes del mundo por su confiabilidad, ya que aporta una dimensión categorizada de la forma en que un individuo aprende dentro de los estilos propuestos por los autores, por consiguiente, el desarrollo de este proyecto permitió hacer una aproximación a una caracterización inteligible del ser humano para estudiarla por medio de una descripción. Este instrumento consta de 20 afirmaciones para cada una de las categorías (Activos o Acomodadores, Reflexivos o Asimiladores, Teóricos o Divergentes y Pragmáticos o Convergentes), para un total de 80 ítems en donde se ha estructurado de una forma aleatoria para que este sea más asertivo. Esta prueba es autoadministrable y se valora por medio de una puntuación dicotómica, es decir de acuerdo (con un signo más + ) o en desacuerdo (un signo menos -), donde se socializo esta estructura con cada una de las estudiantes. 
Como primera fase de la aplicación, se socializó los parámetros del cuestionario CHAEA en los dos horarios de clase pertenecientes al módulo de desarrollo y fue aplicado en el espacio virtual (Aulas virtuales de la Corporación Universitaria Minuto de Dios UNIMINUTO) de cada grupo.
Por otra parte, los resultados fueron estructurados bajo una escala tipo Likert, que consta de 5 ítems que se definieron por muy bajo, bajo, moderado, alto y muy alto; donde cada estilo de aprendizaje se categoriza por la cantidad de respuestas positivas (ver tabla 2):

Tabla 2: Parámetros de clasificación de los estilos de aprendizaje segmentado en escala Likert

\begin{tabular}{|c|c|c|c|c|c|}
\hline ESTILO & Muy Bajo & Bajo & Moderado & Alto & Muy Alto \\
\hline Activo & $0-6$ & $7-8$ & $9-12$ & $13-14$ & $15-20$ \\
\hline Reflexivo & $0-10$ & $11-13$ & $14-17$ & $18-19$ & 20 \\
\hline Teórico & $0-6$ & $7-9$ & $10-13$ & $14-15$ & $16-20$ \\
\hline Pragmático & $0-8$ & $9-10$ & $11-13$ & $14-15$ & $16-20$ \\
\hline
\end{tabular}

Al finalizar la prueba, se valoró cada sección de acuerdo a la orientación del cuestionario donde se procedió a estructurar cada estilo de aprendizaje destacado en cada una de las participantes, posteriormente se dio inicio al análisis de datos, la discusión y las conclusiones respectivas contrastándolas con la valoración académica de los espacios correspondientes a los dos grupos del módulo de desarrollo. Para ello, se realizó la caracterización de la población detallando las variaciones, correlaciones y dispersiones de los resultados obtenidos por medio del software especializado IBM SPSS Statistics, donde fueron organizados los resultados bajo las medidas de tendencia central (media, moda y mediana) para observar el estilo predominante en la muestra del programa. Los datos de caracterización de la muestra y las valoraciones obtenidas en los espacios académicos pertenecientes al módulo de desarrollo fueron analizados por medio de las correlaciones bivariadas, que para este caso se tomó como prueba de análisis las correlaciones de Pearson para observar la significancia entre las categorías y las valoraciones académicas de la muestra, donde se procedió a realizar el análisis y las conclusiones sobre los resultados obtenidos.

\section{Resultados y discusión}

En la presente investigación se pretendía indagar sobre las posibles relaciones entre los estilos de aprendizaje y las valoraciones académicas, por parte de las estudiantes de V semestre de la carrera de Licenciatura en Pedagogía Infantil de la sede principal de UNIMINUTO, lo anterior con el fin de aproximarse a reflexiones que conlleven a mejoras en las estrategias de enseñanza y en el aprovechamiento de los potenciales de trabajo.

La toma de resultados de la muestra, comienza caracterizándose por ser un grupo joven debido a que presentan una media aritmética de 21,79 años con una moda de 24 años donde la mayoría pertenece al estrato 2 . La toma de resultados de la muestra de las estudiantes de LPIN en el cuestionario CHAEA dio una gran apertura a los estilos de aprendizaje que se resaltaban en estos grupos, describiendo una tendencia marcada en las categorías definidas, donde probablemente se destaquen por el perfil del profesional que se está forjando. La categoría de estilo de aprendizaje que se resaltó en promedio de la muestra y con bastante margen de diferencia sobre las otras fue la de Reflexivas o Asimiladoras, puesto que se obtuvo 
una media aritmética de 10,17 con una mediana de 11, es decir que las características de los estilos de aprendizaje de la gran mayoría de estas estudiantes se centran en captar la experiencia abstractamente, juzgan la información mediante un análisis detallado tratando de tener una seguridad completa en esta, para así obtener el conocimiento. La categoría que es menos predominante en este grupo es la de Pragmáticas o convergentes, obteniendo un promedio de 7,72 con una mediana de 8 , donde esta se describe como las personas que prueban sus teorías poniéndolas en práctica, relacionándola con la decisión, donde deben comprobar la información por sí mismos y les gusta trabajar en equipo, pero se les dificulta trabajar individualmente, ya que no se destacan por ser callados o estar quietos.

De acuerdo con los objetivos planteados para la investigación, en los cuales se plantea la necesidad de identificar los estilos de aprendizaje de las estudiantes, en la tabla 3 , se muestra la distribución porcentual correspondiente a los datos del CHAEA:

Tabla 3: Tendencia de estilos de aprendizaje

\begin{tabular}{|c|c|c|c|c|c|}
\hline $\begin{array}{c}\text { Estilos en } \\
\text { muestra total }\end{array}$ & Muy Bajo & Bajo & Moderado & Alto & Muy Alto \\
\hline Activo & 22,1 & 16,9 & 49,4 & 7,8 & 3,9 \\
\hline Reflexivo & 46,8 & 28,6 & 22,1 & 2,6 & 0,0 \\
\hline Teórico & 20,8 & 31,2 & 39,0 & 6,5 & 2,6 \\
\hline Pragmático & 55,8 & 23,4 & 16,9 & 1,3 & 2,6 \\
\hline
\end{tabular}

El estilo activo moderado está presente en un $49,4 \%$, este se caracteriza por personas que se inclinan por estrategias que impliquen constante movimiento, prefieren vivir las experiencias de aprendizaje para hacer efectivo su trabajo, asumen retos y les gusta actuar en grupo, dejando en segunda instancia el reflexionar, por lo cual en ocasiones se les dificulta estar en una actitud pasiva, prolongar los tiempos de labores rutinarias y en solitario.

En segunda instancia, se encuentra que las estudiantes se inclinan por el estilo teórico moderado en un $39 \%$, esto indica que dentro de la muestra existe un número representativo de personas que les gusta aprender de forma metódica, racional y se apegan a las teorías y textos de estudio para analizarlas, trabajarlas y procesar la información de forma lógica para ellos. Por otra parte, se les puede dificultar actividades de aprendizaje que impliquen los aspectos mencionados en el estilo activo, puesto que prefieren comprobar sus aprendizajes al contrastar con asertividad, racionalidad y objetividad.

Otros hallazgos se relacionan con las tendencias muy bajas y bajas existentes en la muestra por los estilos reflexivos con un $75,4 \%$ y pragmáticos en un $79,2 \%$, llevando a percibir que hay poca predilección por la observación, reflexión, análisis, el trabajo enfocado a profundizar y desarrollar pensamiento crítico sin escatimar detalles, en relación a la practicidad que caracteriza a los pragmáticos la muestra no evidencia gusto por comprobación de teorías, la improvisación y el iniciar proyectos en los cuales van aprendiendo a medida que van haciendo. La representación de los estilos en niveles alto y muy alto es escasa, a excepción del estilo activo que es el que se desataca en la muestra.

Ahora bien, al analizar los resultados obtenidos 
en el presente trabajo, se halló que los estilos de aprendizaje preferentes por las estudiantes son de nivel moderado activo con un $49,4 \%$, aspecto que concuerda con García et al (2012), en segunda instancia el estilo teórico con el $39 \%$ y que se usan con mayor frecuencia las estrategias de codificación con un 63,20 y adquisición con un 49,40 concordando con Camarero et al (2000), quien encuentra el estilo activo prevaleciente en la carrera de Magisterio, así como el uso de la escala de adquisición y codificación por la naturaleza del programa, sin embargo, la escala de recuperación presenta una representación fundamental en la muestra.

Por otra parte, la valoración de los espacios académicos del grupo de las estudiantes analizadas, fueron detalladas a través de las medidas de tendencia central, donde se encontró que la media aritmética de la muestra en los espacios académicos es de 37,65 sobre 50 , con una mediana y una moda de 38 , generando una eficiencia del 75,3\% sobre los espacios académicos pertenecientes al módulo de desarrollo.

Finalmente, al detallar la relación entre los estilos de aprendizaje y las valoraciones cuantitativas de los espacios académicos del módulo de desarrollo, se tomó en cuenta la herramienta de correlaciones bivariadas, que en este caso fue a través de la correlación de Pearson, que destaca la significancia entre las categorías de una variable (estilos de aprendizaje) versus otra (valoraciones académicas). Las correlaciones que fueron más significativas sobre las categorías fueron en primer lugar con el estilo de aprendizaje Reflexivas o Asimiladoras, con una significancia del 0.003 sobre 0.01 , es decir que para esta categoría tienen una relación muy próxima con una probabilidad de error del $1 \%$, prácticamente afirmando que las valoraciones obtenidas por las estudiantes de LPIN están muy ligadas a esta categoría. En segunda instancia, se observó que la categoría Pragmática o convergente obtuvo una significancia de 0.032 con respecto a la correlación con las valoraciones académicas, haciendo que tengan también una concordancia bastante cercana según el test de Pearson, resaltando que existe una analogía próxima entre las categorías de los estilos de aprendizaje y la valoración académica en estos grupos de estudiantes de LPIN de la corporación universitaria Minuto de Dios. A pesar de que la correlación es muy notoria con las categorías Reflexivas o Asimiladoras y Pragmáticas o convergentes, las demás categorías presentan una relación muy próxima a los valores óptimos de significancia (0.05), generando una concordancia entre las dos variables.

La concordancia entre las dos variables, para esta situación y contexto se entiende como la relación que existe entre los diferentes estilos de aprendizaje (para este caso fueron segmentados bajo la teoría de Honey \& Alonso, 1994) y las valoraciones obtenidas por varios grupos de estudiantes de la Licenciatura en pedagogía infantil de la facultad de educación de la Corporación universitaria Minuto de Dios UNIMINUTO, donde se evidenció una tendencia en promedio por el estilo de aprendizaje Reflexivo sobre los demás estilos. Para el caso de las estudiantes que desarrolla más este estilo (reflexivo) en particular se evidenció, que obtuvieron un mayor desempeño académico en la valoración final del módulo de desarrollo, evidenciando que para este caso y en este campo del conocimiento, se genera una ventaja para las personas que desarrollan este estilo de aprendizaje en particular. Así mismo, se logró observar que, aunque se presente una escala marcada dentro de un estilo de aprendizaje (muy bajo en el estilo reflexivo $46.8 \%$, muy bajo en el estilo pragmático $55.8 \%$ y moderado en el estilo activo), el promedio de la muestra puede enmarcarse como proporcional (directa o inversa) a la valoración académica obtenida de forma grupal. 


\section{Conclusiones}

Como cierre en el desarrollo de esta investigación se procedió a dar solución a la pregunta de investigación, cumpliendo con los objetivos planteados en el desarrollo de este proyecto abstrayendo las conclusiones más significativas, las cuales fueron:

1. Existe una relación más cercana entre algunos estilos de aprendizaje y la valoración académica del módulo de desarrollo en las estudiantes de LPIN de la Corporación universitaria Minuto de Dios. Frente a ello, se logra divisar la necesidad de reflexionar sobre el entramado que se obtiene al interceptar las estrategias metodológicas, las formas de evaluación en el aula, las competencias que se pretenden desarrollar y el perfil general del pedagogo infantil, con el fin de enriquecer la discusión en torno a la formación docente y las diferentes aristas que se despliegan en la construcción de una propuesta educativa desde la diversidad.

2. Los grupos con los que se trabajó el proyecto se caracterizaron por desarrollar en su gran mayoría dos tipos de estilos de aprendizaje que probablemente se apropien por el perfil profesional que están entablando. $\mathrm{Al}$ respecto, se debe recordar que los estilos marcan tendencias que pueden variar en concordancia con las situaciones contextuales y ello implica que las condiciones laborales pueden potenciar capacidades que se presentan en niveles bajos en un plano de formación, razón por la cual las prácticas profesionales deben propender por la diversidad de escenarios y situaciones que faciliten la exploración de otros estilos.

3. El desarrollo de los estilos de aprendizaje, probablemente, se van potenciando acorde al perfil profesional que se estructure en los estudiantes, generando una construcción cognitiva que en la mayoría de casos puede visualizarse a través de las valoraciones académicas. Sin embargo, la nota como único referente se convierte en un elemento descontextualizado al desligarse del plano colectivo, es decir, la formación del docente debe entrar en dinámicas de evaluación conjunta de las competencias adquiridas y de procesos que impliquen la valoración de capacidades diversas que permitan entrar en diálogos con la multiplicidad presente en la educación de las infancias.

4. La tendencia que existe entre estrategias y habilidades que son propias, de alguna forma limita algunos procesos de aprendizaje en los estudiantes y en ocasiones podría afectar la motivación que se tiene hacía un espacio académico, generando la necesidad de indagar más sobre los estilos y estrategias predominantes en los estudiantes, con el fin de generar un equilibrio en las estrategias metodológicas utilizadas dentro del aula. En este sentido, se evidencia la necesidad de generar marcos de participación y reflexión sobre la construcción y adecuación de los micro currículos o syllabus de los diferentes espacios académicos y que la voz de los estudiantes se haga presente en los mismos, pues de esta forma se da paso a la comprensión de los procesos de enseñanza y aprendizaje en un marco de la diversidad.

5. Se debe incentivar el uso de proyectos de trabajo, estudios de caso o aprendizaje basado en problemas que impliquen el uso de los diferentes estilos de aprendizaje, estrategias de aprendizaje en el desarrollo de los mismos y que coloquen en juego las fortalezas y potencien las debilidades de los estilos de aprendizaje predominantes de los participantes del grupo. Para el caso 
de módulo de desarrollos las metodologías no deben situarse en el plano teórico como única opción, pues este constituiría el referente desde el cual se crean los ambientes de aprendizaje que potencien las capacidades innatas de las infancias, aspecto que combina los diferentes estilos al requerir la apropiación de los fundamentos conceptuales para la construcción de propuestas pedagógicas que se basen en la observación, el análisis de contexto, la planificación, la ejecución y la reflexión sobre lo sucedido en las intervenciones. De otra parte, la labor del pedagogo infantil implica establecer lazos de cooperación con otros profesionales (terapeutas, fonoaudiólogas, psicólogos, trabajadoras sociales, entre otros) con el fin de comprender las diferentes realidades que se presentan en los contextos que habitan las infancias, por lo cual, se deben proporcionar estrategias de simulación y análisis con el fin de ampliar la mirada disciplinar que se presenta en el desarrollo infantil y pasar a una perspectiva interdisciplinar y transdisciplinar que brinde opciones desde el accionar del docente.

\section{Referencias}

Aguilera, P., Ortiz, T. (2009). Las investigaciones sobre los estilos de aprendizaje y sus modelos explicativos. Revista Estilos de Aprendizaje, 4. 22-35 Recuperado de http://www.uned.es/revistaestilosdeaprendizaje/numero_4/Artigos/lsr_4_articulo_2. pdf

Aguilar, R. (2010). Estilos y estrategias de aprendizaje en jóvenes ingresantes a la universidad. Revista de Psicología. 28 (2) 207-225. Recuperado de http:// www.redalyc.org/articulo.oa?id=337829515001

Arnaiz, P. (2003). Educación inclusiva: una escuela para todos. Málaga: Aljibe.

Hojas y Hablas ISSN (e) : 2539-3375 № 14. Año 2017. [Pp. 131-142]
Alonso, C.; Gallego D.; Honey, P. (1994). Los Estilos de Aprendizaje: Procedimientos de diagnóstico y mejora. Bilbao: Ediciones Mensajero.

Bustos, S. L. E. (s.f). La evaluación: Una mirada desde los estilos de enseñanza y de aprendizaje. En Observatorio Nacional de Políticas en EvaluaciónONPE, Políticas y experiencias de evaluación de la Educación Superior en Colombia. 183-196). Bogotá: Editorial Universidad Pedagógica Nacional.

Camarero, S. Martín del Buey, F, Herrero. Diez, J. (2000). Estilos y estrategias de aprendizaje en estudiantes universitarios. Psicotherma. 12, (4), 615-622. Recuperado de http://www.psicothema. com/pdf/380.pdf

Cardona, A. (2005). Las capacidades en las competencias laborales: Una mirada desde las Inteligencias múltiples en los jóvenes. Revista Universidad EAFIT. 41 (140). 25-42. Recuperado de http://www. redalyc.org/articulo.oa?id=21514003

De Tezanos, A. (2006). El maestro y su formación: tras las huellas y los imaginarios. Bogotá. Cooperativa editorial del Magisterio.

Esguerra Pérez, G; Guerrero Ospina, P; (2010). Estilos de aprendizaje y rendimiento académico en estudiantes de Psicología. Diversitas: Perspectivas en Psicología, 6. 97-109. Recuperado de http://indicadorescti.gob.mincyt.mincyt.mincyt.redalyc. org/articulo.oa? $\mathrm{id}=67916261008$

Gallego, G., D. J; García, D. (2012). Los estilos de aprendizaje en la formación inicial del docente. Revista Estilos de Aprendizaje, 5 (9) Recuperado de http:// www.uned.es/revistaestilosdeaprendizaje/numero_9/sumario_completo/lsr_9_abril_2012.pdf

García, C., Sánchez, Q., Jiménez, V. Gutiérrez, T. (2012). Estilos de Aprendizaje y Estrategias de Aprendizaje: un estudio en discentes de postgrado. Revista Estilos de Aprendizaje, 10, (10), o c tubre de 2012.

Honey, P. y Mumford, A. (1986). The Manual of Learning Styles. Maidenhead, Berkshire. P. Recuperado el 16-05-2015 de: http://www.alammi.info/ 
documentos/1congreso/memorias/mie2_2.pdf

IBM Corporation. (2011). Guía breve de IBM SPSS statistics 19.0 EE.UU.: Editorial copyringht IBM

Lozano, A. (2006). Estilos de aprendizaje y enseñanza. Un panorama de la estilística educativa. México: Editorial Trillas.

Mendoza Borrero, W. (2012, junio). Los estilos de aprendizaje en estudiantes Universitarios: estado del arte y desafíos. Comunicación presentada en Congreso Mundial de Aprendizaje, Santander. Recuperado en http://dialnet.unirioja.es/servlet/ articulo? codigo $=4665796$

Morin, E. (2009). El método 5. La humanidad de la humanidad, la identidad humana. Madrid. Ediciones Cátedra.

Salas Silva, R.E. (2012). Estilos de aprendizaje a la luz de la neurociencia. Bogotá: Cooperativa del Magisterio.

Sánchez, G. Andrade, E. (2014). Inteligencias múltiples y estilos de aprendizaje, diagnóstico y estrategias para su potencialización. México: Alfaomega. Instituto de ciencias de la educación Estado de México. 